\title{
ENFERMEDAD MENTAL Y SOCIEDAD EN LA EUROPA DE LA SEGUNDA MITAD DEL SIGLO XIX*
}

\author{
Raquel Alvarez \\ Rafael Huertas \\ José Luis Peset
}

Es difícil en pocas páginas intentar dar razón del proceso de somatización que la enfermedad mental conoce a lo largo de la segunda mitad del siglo XIX. Lo es más si se tiene en cuenta la diversidad del pensamiento psiquiátrico en cada uno de los países europeos. Sin embargo, no creemos carente de valor procurar explicar este proceso, enmarcándolo en nuestras propias investigaciones, así como en la más actual bibliografía aparecida (1). Habitualmente se barajan dos tipos de explicaciones para este proceso, unas de carácter profesional e institucional y otras de carácter científico y técnico. Las primeras pueden enraizarse en un excelente trabajo del investigador francés G. Lanteri-Laura, quien en 1974 se plantea las causas del fracaso manicomial en su país en la segunda mitad del siglo XIX y las consecuencias médicas a que llevó (2).

* Este trabajo fue presentado en el $17 .^{\circ}$ Congreso Internacional de Ciencias Históricas celebrado en Madrid en 1990. 


\section{Asilos y enfermedad mental}

A partir de mediados de siglo, se produce un cambio en las condiciones de observación clínica, que determinan consecuencias en la consideración etiopatogénica y en sus resultados en el pronóstico y la terapéutica de la enfermedad mental. Los hospitales para alienados que se construyen en la Europa del XIX, son, sin duda, tardíos, edificados por una sociedad burguesa conservadora, que poco tiene que ver con quienes los proyectaron. En consecuencia, el espacio manicomial lleva hacia la cronificación, dados factores tales como el aumento de edad de la población, la insistencia en el control social, la conveniencia para la pervivencia de la misma institución, el desarraigo social, laboral y familiar de los internados, etc. Todo esto lleva hacia una nueva formulación teórica y observacional de procesos y cuadros morbosos, insistiendo el autor que comentamos en la consideración privilegiada del delirio crónico y la demencia, y a un fortalecimiento y pervivencia extremos de la teoría de la degeneración (3). Es evidente que esta búsqueda clínica y teórica también encierra cierta esperanza en la posibilidad de prevención y curación de la enfermedad somática, oscilando por tanto el psiquiatra de fin de siglo entre el pesimismo de la «defensa social» (4) y el optimismo del «remedio específico» y de la «eugenesia» (5).

Todas estas novedades se acompañan de un intento por parte de los médicos por conseguir la administración del enfermo mental. Datos tales como la participación de Esquirol en la ley de alienados de 1838, tras el juicio de Pierre Rivière en 1835, con una brillante pléyade de alienistas a su favor, y la actuación de Lacassagne contra el asesino del presidente. Carnot en 1894, marcan las etapas principales de esta historia, en la que el psiquiatra iba ganando peso ante los poderes ejecutivos, legislativo y judicial, primero en violencia enemiga y más tarde como un cuarto o quinto poder, aliado con aquéllos que la sociedad burguesa iba poniendo en pie (6). En todas estas actuaciones públicas de los médicos, las aportaciones políticas de los profesionales eran semejantes, ofrecer saberes técnicos adecuados al poder central a cambio de su mejora social, legal e institucional.

Este mismo proceso se contempla a lo largo del siglo XIX en Inglaterra: el desarrollo de un sistema asilar caracterizado por la implantación progresiva del non-restraint (7) -incluyendo aquí tanto las formas de control del enfermo agitado, como el tratamiento moral-, la extensión de una cadena de asilos condales, y la regulación legal de organismos y 
autoridades de dirección e inspección de los establecimientos públicos y privados dedicados al cuidado y tratamiento de los insanos. En este camino, la extensión del non-restraint, y de las primeras inspecciones y puestas al día de la situación de los alienados en Inglaterra, llenan la primera parte del siglo, mientras la segunda se caracteriza por ese desarrollo del número de instituciones de que hablábamos, y por el establecimiento de una legislación cada vez más compleja, que regulaba tanto el internamiento, como el trato y el alta de los insanos, así como la forma de dirección de los asilos.

Es importante señalar que todo este proceso, en el que participaban médicos, pero fundamentalmente legisladores, políticos y juristas, se da también en Inglaterra en momentos en que la profesión médica luchaba por conseguir un valor social significativo, comparable al de los juristas (8), pugnando por hacer valer su derecho a tratar, controlar e intervenir en el proceso de reforma asilar en todos sus aspectos. Y es significativo que la profesionalización y sobre todo la especialización del alienista, no se produce en principio por medio de unos estudios o preparación determinada, sino a través del acceso a puestos de superintendente o director de un establecimiento, o de funcionario relacionado con el sistema asilar. Asociaciones y publicaciones tienen, en un principio, nombres que se refieren a esta situación, modificados más tarde con una orientación más especializada: la Asylum Officer's Association cambiará su nombre en 1865 para convertirse en la Medico-Psychological Association.

En 1838 - fecha también importante para Francia- se produce un importante enfrentamiento entre médicos y legistas, cuando el Select Committee on the Poor Law Amendment Act -y seguramente Chadwickquiere poner el sistema de los County Asylums bajo el control de la Poor Law. La única forma de defensa de los asilos como sitio de internamiento de insanos, ineptos, disminuidos y pobres, mucho más caros para el erario público que las workhouses, era insistir en que aquéllos eran un lugar de tratamiento. Tratamiento que suponía «curabilidad», por lo menos en un buen porcentaje. A mediados de siglo todo el mundo, incluso las propias autoridades, aceptaban el éxito del non-restraint, a pesar de algunas voces discordantes (9). Se confía en el tratamiento asilar y en la reinserción gracias al trabajo que allí se realiza. De todas formas, el tratamiento moral, base fundamental de la asistencia, no hacía olvidar por entero el tratamiento médico, si bien sus posibilidades eran por entonces limitadas. Y tampoco dejaba de haber miedos a la reclusión asilar por parte del público, tanto pobre como burgués, cuidando los legisladores de salvaguardar 
la libertad individual y familiar, ante el recelo de los médicos que querían más poder y tratamiento temprano (10).

El panorama cambia, como en Francia, en la segunda mitad del siglo, tal como se refleja en el excelente informe de 1877 de M. Granville, The Care and Cure of the Insane (11). Se señala allí cómo, tras un período de gran actividad, en el que se habían remediado los peores abusos del sistema de manicomios, los asilos se habían estancado y, en algunos casos, incluso se había producido una regresión. También en Hanwell, indica Granville, el trabajo de Conolly, el campeón del non-restraint, había languidecido. Señala como posibles causas de esta situación la falta de dinero -quizá en relación con la crisis de 1875- y también la carencia de contacto personal que existe en los establecimientos. Y expresa la necesidad de un cambio radical de actitud ante el insano. Ya no son suficientes las primeras medidas del non-restraint.

De forma paralela se esbozan nuevas formas de ejercicio de la medicina, en lo que a la profesión y al tratamiento de los enfermos mentales se refiere. Debemos recordar que los años ochenta son también los años de los albores de la lucha por la higiene mental, y que además se señala insistentemente la necesidad, no sólo del «especialista» médico, de la enseñanza específica -que debe asentarse tanto en el estudio clínico, como en la exploración del sistema nervioso- del médico alienista, sino también del attendant, quien trata continuamente con el enfermo. Y junto a la progresiva pérdida de prestigio del asilo, se comienzan a proponer nuevas formas de atención al paciente: tratamientos en cottages, en la propia residencia del médico, sistema de "puertas abiertas», etc. Y se considera - ya se había iniciado esta actitud en 1844- la posibilidad de separar los enfermos curables de los incurables.

En efecto, la disminución del optimismo acerca de la curación del enfermo mental acompaña al pesimismo ante los hospitales, surgiendo la idea de que las instituciones victorianas están atestadas de enfermos crónicos (12). Muchos establecimientos mantenían una especie de comunidad en que los internos se dedicaban a diversas tareas, agrícolas y artesanales, que adquiría tintes de situación establecida. Sin embargo Ray (13) demuestra en sus estudios de los libros - case-notes - de los asilos, llevados religiosamente desde las leyes de 1845 , que esta situación de internamiento prolongado no era tan frecuente, sino que lo era más el ingreso y alta - por diversas razones, incluso traslados- al menos antes de diez años, pero en general dentro de los dos primeros años de internamiento. Afirma que el turnover de enfermos era muy intenso y que así se mantuvo 
hasta los años noventa. Pero el propio Ray señala cómo la imagen del insano crónico se hace prominente en la teoría psiquiátrica y también en su práctica. La insania, dice Ray, deja de ser una etapa en la vida de alguien, una "enfermedad», para convertirse en una «incapacidad" permanente.

Si ya en 1844 y de nuevo en 1867 se estableció el principio de la separación de curables e incurables, en 1881 la Medico-Psychological Association seguía, sin embargo, detrás de esta separación. En términos generales, esta denominación de incurables se refería a epilépticos, idiotas y otros deficientes mentales, aunque también podían incluirse insanos inofensivos. Posteriormente se planteará la necesidad de la separación para abaratar costes de mantenimiento de estas personas, pues se propone un régimen como el de las workhouses, mucho más barato, para los crónicos o incurables, puesto que los asilos, aunque los internos trabajen, no consiguen autofinanciarse. Muchos médicos están, además, a favor de la separación, por las ventajas que podría traer para el tratamiento, e incluso porque ello permitiría una mayor diversificación de la forma y sitio del tratamiento. Eran nuevas formas, fuera del asilo masificado tradicional.

Para España Josep María Comelles ha desarrollado esta tesis, mejorándola desde el punto de vista de la historia profesional, insistiendo en el papel que el manicomio juega como instrumento del psiquiatra. Es evidente que el médico alienista colocó el manicomio como instrumento esencial de su prestigio profesional. Y también lo es que la simbiosis entre profesional e institución llevó a la larga al desprestigio del médico alienista. Si bien el manicomio surge para el empleo del "aislamiento" (dentro del "tratamiento moral») para la curación de las enfermedades, no hay duda de que al aislar al enfermo buscaban varios objetivos. Por un lado, un intento de tranquilizar al paciente, a sus familiares y a la sociedad; por otro, el romper los lazos con el medio habitual, considerado nosógeno con frecuencia. En tercer lugar, reconstruir su vida, rehaciendo sus lazos referenciales, de tipo familiar en primer término.

\section{La somatización de la locura}

Resulta fundamental insistir en que el tratamiento moral tiene razón de ser en tanto se considere al loco un sujeto recuperable. Para F. Leuret, «la folie consiste dans l'aberration des facultés de l'entendement; elle n'est pas, comme les maladies ordinaires, caractérisées par des symptômes physiques, et les causes qui la produisent, quelquefois appréciables aux 
sens, appartiennent la plus souvent à un ordre de phénomènes complètement étrangers aux lois générales de la matière: ce sont des passions et des idées (14)».

Esta concepción "psicologista» de la locura, justificadora de las más duras medidas «morales» intimidatorias que pudiesen sacar al loco de su "error», es el soporte del tratamiento moral. Esta forma de terapia, con la que la psiquiatría se despega de las actuaciones propias de la medicina general, es la consecuencia de una concepción «moral» de la enfermedad mental que es predominantemente psicogenética y social, pero sin abandonar jamás, como ya dijimos, la base fisiológica ni los remedios médicos, que permitían actuar en el frágil nexo entre fisiología y moral. En cualquier caso, el que la locura estuviera presa en un mundo moral implicaba una postura conservadora, que tuvo su contrapartida en las corrientes somaticistas que a partir de la segunda mitad del siglo comenzaron a aparecer cada vez con más fuerza.

Por ello es necesario valorar en su justa medida estas corrientes, porque la mayoría de los psiquiatras organicistas fueron, en un principio, médicos progresistas que, comprometidos políticamente con la revolución del 48, plantearon las primeras críticas al manicomio y al tratamiento moral, siendo en muchas ocasiones reformadores profundos de las instituciones. Es éste un buen ejemplo de cómo a lo largo de la historia las posturas "psicologistas" $\mathrm{y}$ "somaticistas» han jugado papeles ideológicos diferentes (15). Paradojas de la ciencia, como la que nos muestra a ese movimiento somaticista esperanzador al principio, cuando quiso evitar la tortura y la intimidación, pero reaccionario a la larga, pues al colocar al loco en el absoluto determinismo que la ciencia positiva predicaba, hizo que el pesimismo antropológico y el fantasma de la incurabilidad planearan sobre los manicomios, sumiéndolos en el más lamentable abandono. Se dejó de torturar e intimidar sistemáticamente a los pacientes, a cambio de considerar a una gran mayoría de ellos como irrecuperables y, por tanto, susceptibles de encierro permanente.

El tratamiento moral había fracasado, pero el manicomio como institución total (16) se mantuvo con una muy devaluada vocación terapéutica y con el claro reforzamiento de su papel de control y defensa social. Por ello es evidente, como señala Comelles para España, que el manicomio se convirtió en una anticuada explotación agrícola o artesanal, con enfermos crónicos cada vez más envejecidos y en donde el custodialismo fue el elemento fundamental. Paralelamente se produjo el proceso de somatización de la enfermedad mental (17), pues el fracaso 
manicomial se enlazó con la consideración somática de la enfermedad mental, que se manifestó en diversas formas. Hay una orientación clínical fácil de observar, que fue la orientación neurológica que la psiquiatría de la época vivió. La neurología, ciencia de moda a fines del XIX y principios del XX, permitió una clínica muy objetiva, con pruebas exploratorias muy rigurosas y unos diagnósticos que presentaban una gran convicción. Aparte, esta aproximación a la neurología permitió también el acceso de los alienistas a otros espacios, sea el gabinete especializado, sea el laboratorio, sea la sala de autopsias. El manicomio dejaba de ser el peligroso amigo incondicional, que había sido por muchos años para el psiquiatra (18). Es evidente que otros aliados se aproximaban, así los mármoles de las salas de disección, en donde se buscaban y localizaban causas somáticas de enfermedad, casi con exclusividad en el sistema nervioso.

Es evidente que había una larga tradición observacional que unía la psiquiatría con la alteración del sistema nervioso, que se muestra ya en las dudas de Philippe Pinel por interpretar o no la enfermedad mental como consecuencia de una lesión neurológica. Al llegar las alturas del ochocientos una muy abultada colección de hallazgos necrópsicos avalaban una intelección somática de la enfermedad mental. Pero todavía, en el cambio de siglo, el Pinel del Traité y el Esquirol del Des passions (19) dudaban de la asignación de última causa a las lesiones morfológicas. Pero a partir de la publicación en 1838 de los dos volúmenes del tratado Des maladies mentales de este último autor, las cosas han cambiado. Con ello, con la aceptación de la causa morfológica como primordial, no se hacía sino recoger una ya antigua tradición médica y clínica. Desde la aparición de la obra de Morgagni, el médico patólogo buscó con creciente interés la localización de la enfermedad. Primero se consiguió en órganos enfermos, a partir de las lecciones del italiano, luego en los tejidos patológicos, a través de la labor del francés Bichat, y por fin en las células, con los logros del alemán Virchow (20). Con el tiempo, gracias en buena parte a los trabajos de Santiago Ramón y Cajal sobre la organizaicón del sistema nervioso, la enfermedad se relacionará también con las alteraciones de la estructura y organización del tejido neuronal — textura en términos cajalianos (21) - . Naturalmente, el camino recorrido en el terreno de la fisiología entre F. Magendie y C. Bernard será fundamental para hacer derivar la lesión de la estructura de los órganos, como el cerebro (22).

Si los primeros alienistas expresaron sus observaciones clínicas a través de clasificaciones nosográficas more botanico —que, siguiendo la 
pauta de Sydenham, se basaron en la observación y ordenación de la sintomatología de cientos de enfermos (23) - , en la segunda mitad del XIX esta semiología fue dando paso a una insistente búsqueda de la etiología de las enfermedades mentales. Así, el degeneracionismo intentará encontrar las causas del inusitado aumento de la morbilidad psiquiátrica y de la masificación de los asilos que tiene lugar en los años centrales del siglo. Pormenorizadas listas de causas degeneratrices, entre las que destacan la herencia y la acción sobre el organismo de determinados agentes tóxicos, como el alcohol, empiezan a ser postuladas con insistencia como factores etiológicos de la locura en un discurso psiquiátrico, que pretendió y consiguió afianzar la concepción somática de la enfermedad mental. La formulación definitiva de la teoría de la degeneración, llevada a cabo por V. Magnan y P. M. Legrain no deja lugar a dudas: «Le terme dégénérescence appliqué à la pathologie mentale désigne l'état morbide d'un sujet dont les fonctions cérébrales accusent un état d'imperfection notoire, si on les compare à l'état cérébral des types générateurs (24)». Patología constitucional y hereditaria, causas físicas, localización cerebral, etc. eran también formas de soslayar, bajo pretendidas razones científicas, los evidentes motivos sociopolíticos y económicos que intervinieron directamente en la génesis del alcoholismo, la criminalidad y no pocas formas de enfermedad mental a lo largo de la segunda mitad del siglo XIX.

No cabe duda de que la teoría de la degeneración responde a la inquietud de los psiquiatras positivistas por encontrar las causas de la alienación mental, por contar con una explicación etiológica de la locura. Como apunta Robert Castel, en un primer momento, cierto número de alienistas se esforzaron por escapar de los dilemas entre causas morales y causas orgánicas, entre descripción de los síntomas y búsqueda de la radicación, para llegar al conocimiento de la enfermedad en función de su evolución y no de sus manifestaciones clínicas. De este modo, Lasègue aisla el delirio de persecución en 1852. Falret y Baillarger describen simultaneamente lo que uno llama "locura circular» y el otro "locura de doble forma». Estos autores no se contentan con describir un síntoma o un conjunto de síntomas sino que, en un intento de aislar entidades nosográficas, hacen de cada síntoma un signo que, junto a otros, permiten un mejor conocimiento de los procesos patológicos mentales. Se pasa así de una sintomatología como simple proceso descriptivo, a una semiología por la cual la enfermedad adquiere a la vez un sentido subyacente en sus manifestaciones externas y un potencial evolutivo (25). 
Con la teoría de la degeneración se da un paso más, de la semiología a la etiología, lo cual trae consigo una consecuencia inmediata: si se saben o se intuyen las causas de la locura o, lo que es más trascendental, si se pueden diagnosticar alienaciones en potencia -mediante la identificación de las causas degeneratrices (herencia, intoxicaciones, enfermedades congénitas o adquiridas...) - , los médicos, y la sociedad, podrán poner los medios adecuados para evitarla o prevenirla. Por otro lado, la frenología predica también el progresivo abandono del estudio de la mente a través de la metafísica y sus técnicas introspectivas, y el paso hacia el estudio del cerebro y su fisiología como asiento de las «facultades», después «funciones» mentales (26). El problema teórico se centraba, fundamentalmente, en las relaciones mente-cuerpo, que buscaba determinar hasta qué punto podía progresarse en el conocimiento del ser humano, y de su comportamiento normal o anormal, utilizando una u otras formas de aproximación. Las ideas de Gall prendieron con rapidez en todo el continente e incluso en Inglaterra. Así la importancia de la fisiología del sistema nervioso como base del funcionamiento de la mente fue en aumento a lo largo del siglo, y puede decirse que en su segunda mitad había una aceptación generalizada de esta base orgánica, así como de la importancia de las alteraciones del cerebro en relación con las causas de la enfermedad mental (27).

- Tal como escribe Jacyna (28), en los años cuarenta y cincuenta se produjeron grandes cambios en la neurociencia inglesa, con la extensión de la actividad refleja, ya comprobada a nivel de médula espinal, al sistema nervioso central. Estudiosos venidos en muchos casos de la filosofía, como Alexander Bain, integraron las nuevas corrientes del pensamiento - positivismo, asociacionismo- con los nuevos conocimientos fisiológicos (29), corriente seguida por alguno de los alienistas ingleses de renombre, como Henry Maudsley. Si no todos apoyaban esta concepción unitaria (30), sí fue general la aceptación de una ineludible relación entre la mente y el cerebro, reforzada por esos conocimientos en expansión sobre el funcionamiento reflejo del cerebro y sobre la localización de las funciones (31). El evolucionismo spenceriano, así como el darwinismo, influyeron grandemente en cuanto a la importancia de la relación entre el organismo y el medio, el estudio de los diversos niveles de organización y la aceptación de la experimentación animal como significativa para el conocimiento del sistema nervioso humano.

En su discurso a la Medico-Psychological Association, en 1881, afirmaba $\mathrm{D}$. H. Tuke que en los últimos cuarenta años se había producido un 
gran cambio en el reconocimiento de la enfermedad mental como parte integral de los desórdenes del sistema nervioso, y que la psicología médica era considerada cada vez menos como un fragmento desgajado del dominio general de la medicina (32). Uno de los textos psiquiátricos de mayor difusión durante la segunda mitad del XIX, A Manual of Psychological Medicine de Bucknill y Tuke, cuya primera edición es de 1858, y la cuarta de 1879 , afirma ya: «The broad view of the production of Insanity appears to be this: The brain, like every other organ of the body, for the perfect performance of its functions requires the perfect condition of its organization, and its freedom from all pathological states whatever. Consequently, the existence of any pathological state in the organ of mind changes its healthy functions, and produces a greater or less amount of disease of mind - that is, of Insanity-». Y también indican estos autores: "The great principle that Mental Disease depends solely upon cerebral conditions, which was systematically taught in these pages fifteen years ago, has now become so thoroughly established that is no longer questioned (33)».

En España se produjo un avance semejante, con importante repercusión en la psiquiatría, pero las novedades vinieron más de la histología que de la fisiología. La «escuela histológica española» encabezó el movimiento de renovación científica de la segunda mitad del siglo XIX, centrando pronto su atención en la morfología normal y patológica. De todas formas, Cajal estuvo siempre obsesionado por el funcionamiento de la mente, el origen del pensamiento humano fue tema que siempre le ocupó, si bien al principio fueron las armas empleadas en este estudio la psicología experimental y el hipnotismo, pronto el contacto con Luis Simarro le llevó al conocimiento de la escuela de Bernard y a los más modernos métodos histológicos (34). No es por tanto extraño que estableciera todo un programa de investigación anatomopatológica para hallar las causas de la alteración del funcionamiento de la mente en las lesiones cerebrales.

Sus palabras son muy claras: «No existe alteración funcional sin lesión anatómica, como no hay lesión sin causa exterior perturbadora, conocida o desconocida. Suponer la existencia de enfermedades sin lesión, es tanto como afirmar que las manifestaciones fisiológicas pueden cambiar caprichosamente, escapando a todo determinismo científico. Los autores que, por no haber hallado perturbaciones anatómicas en la autopsia, describen enfermedades sin lesión, olvidan que, bajo la máscara de una normalidad exterior, pueden ocultar los tejidos graves alteraciones microscópicas, las cuales no son siempre reveladas con nuestros recursos analíticos, porque distamos mucho de haber descubierto todos los delicadísimos resortes de 
la estructura celular y todas sus posibles desviaciones. A medida que nuestros medios de observación se perfeccionan, más se va reduciendo el número de enfermedades sin localización, pudiendo afirmarse que cada proceso notable en la técnica se traduce por la reducción a perturbaciones anatómicas concretas de algún proceso reputado irreductible. Y aún suponiendo que nuestros recursos amplificantes nos permitieran agotar el orden morfológico, quedaría aún, con todas sus oscurísimas incógnitas, el orden químico normal y perturbado (35)». Si bien el programa de investigación no era nuevo, pues continuaba una antigua tradición, tal como hemos afirmado, sí resultó de gran interés porque se aplica al sistema nervioso. Las mejoras técnicas y el estudio de algunas patologías - como las placas seniles cerebrales- permitieron a Cajal encabezar una notable «escuela española» que en anatomía patológica del sistema nervioso consiguió muy notables aportaciones, así al estudio de la rabia, la epilepsia o la demencia senil. Autores como Achúrraco, Río-Hortega y Rodríguez Lafora llevaron adelante en el primer tercio del siglo XX los programas anatomopatológicos de la escuela.

Paralelamente, este interés en hallar causas somáticas a la enfermedad mental, entró pujante en psiquiatría, sin duda por influencia del degeneracionismo francés. Superado el primer "psicologismo» de Pi i Molist, la escuela psiquiátrica catalana - de clara influencia francesa y de orientación positivista y organicista - tiene, primero en Giné i Partagás, y más tarde en su discípulo Galcerán, sus más destacados representantes. La obra de este último supone, sin duda, la culminación de la corriente somaticista. «La naturaleza de la locura - escribe- queda determinada por la de la razón. Ambas son modos de funcionar del cerebro y la vida de éste es un fenómeno químico-mecánico (36)». La enfermedad mental entendida como «cerebropatía» está presente en el pensamiento anatomoclínico de este autor, quien se muestra esperanzado en que la anatomía patológica consiga tarde o temprano, dar respuesta a los interrogantes que las causas de la locura seguían planteando (37).

\section{Criminales o locos}

Pero todavía hay otra razón importante para el proceso de somatización que la enfermedad mental conoce en la últimas décadas del siglo XIX. Se trata de la vieja relación entre psiquiatría y medicina legal, pues las mismas cátedras explicaban en algunos países las dos disciplinas y los 
psiquiatras eran llamados desde tiempo atrás a los tribunales. Su potenciación mutua, hizo que los profesionales que acudían al manicomio y al foro se habituaran a unir campos científicos y semánticos de peligrosa vecindad. El médico y su público se acostumbraron a unir delito, enfermedad y lesión anatómica. Y si de esta unión resultó prestigio e influencia para la clase médica, también supuso un avance en el camino hacia la cronicidad y la somatización de la enfermedad mental. Si bien en un principio se pensaba que de esta unión iba a resultar una mejora para el delincuente enfermo, a la larga supuso un peligro que condujo hacia la «defensa social» y el «lombrosismo». Era un camino de larga tradición, que se heredaba de viejos contactos de la medicina con el mundo del pecado y la brujería. Ahora, con la secularización de la sociedad, al pecado lo sustituye el delito, pero con cierta carga negativa tal como la enfermedad mental siempre ha tenido. Carga que irá hacia la somatización, así cuando Morel hace derivar la degeneración del pecado original (38).

No es extraño, por tanto, que el italiano Cesare Lombroso recoja la tradición médico-legista, la anatomopatológica y las teorías de la degeneración. Para él el delincuente -que identifica al enfermo mental-es un salvaje que ha quedado en una sociedad avanzada. Las razones de esta alteración puede explicarlas desde el degeneracionismo, o bien desde el evolucionismo, como sucederá a lo largo de su obra. Pero es evidente que pondrá al servicio de la «defensa social» tanto estas sabias teorías, como un detallado estudio del cuerpo humano, que permitieran la identificación de sujetos considerados peligrosos. Con ello la medicina legal y la psiquiatría conseguían un perfecto hermanamiento con la sociología y la jurisprudencia - gracias a su colaboración con Ferri y Garófalo- y la enfermedad, el delito y la lesión se confundían. Así contribuían de forma poderosa a ese proceso de somatización de la enfermedad mental, con olvido grave de otras consideraciones de tipo sociológico (39).

El «lombrosismo» fue un pensamiento de gran difusión, pues llegó tanto a Europa como a América, influyendo en áreas tan distintas como la literatura, la medicina, el derecho o la sociología. Sin embargo, en cada país y en cada disciplina penetra con peculiaridades propias. Esta diversificación - por otro lado, normal en la difusión de las ideas- se acentúa por el hecho de que el mismo Lombroso - y su escuela- conoció una clara evolución en su pensamiento. Tras una primera etapa, en que se centra en la idea del criminal nato, como individuo retrasado respecto a la evolución de la sociedad, las críticas fueron enormes. Se reconocía esta idea como sugerente - se añadía a las novedades darwinianas- pero sin explica- 
ción causal ni fisiológica. El italiano se ve obligado a explicar por diversas teorías médicas la aparición de este "salvaje» en la sociedad civilizada y las buscó en las ideas de moda. Por un lado, tomó prestada la decadencia degeneracionista, por otro la debida a diversas enfermedades o agentes nosógenos, en especial la epilepsia. Por su lado, el sociólogo Ferri insistió en los aspectos sociológicos y el jurista Garófalo en los penales.

En Francia se difundió ampliamente el pensamiento de Lombroso, si bien siempre desde el punto de vista crítico. Era lógico que desde la patria del degeneracionismo se viera como cosa propia las ideas de este autor, si bien con autoridad suficiente para modificarlas. Muchos comentaristas subrayaron, quizá de forma especial en el campo psiquiátrico, el papel de la degeneración en la formulación del concepto de criminal nato. Así, en contra de la explicación atávica del crimen, propia del lombrosismo temprano, que el filósofo Tarde había criticado, la escuela francesa se pronunció en repetidas ocasiones. Para Ch. Féré, la criminalidad debía ser considerada tan sólo como una forma inferior de degeneración, asegurando que «l'hérédité criminelle est une variété de l'hérédité dégénérative» y, en contra de la teoría lombrosiana del criminal nato, "le criminel ne représente pas un type atavique distinct du dégénéré (40)». De esta misma opinión será Magnan, quien tras reconocer el gran mérito de la escuela italiana al haber considerado la criminalidad en el campo antropológico y de acometer el estudio biológico del delincuente, revisa críticamente los puntos fundamentales del lombrosismo, para concluir afirmando que «le criminel né n'existe pas, ou, s'il existe, c'est un dégénéré (41)».

Mas fiel al maestro italiano, al que llama "mon ami M. Lombroso", y cuya clasificación retoma con fidelidad, es el médico-legista A. Lacassagne. Quizá su dedicación forense lo justifica, quizá se deba a su aceptación de las explicaciones tardías de Lombroso, sea el degeneracionismo, la epilepsia, o una mala evolución (42).

También se produjo en Inglaterra la entrada de las ideas lombrosianas -así como en América-, que encajaban muy bien con las ideas evolucionistas de la época. Pero no menos se tiene en cuenta a la escuela degeneracionista, cuyo influjo en todo el continente era decisivo como apoyo al psiquiatra italiano. Por ello, en Inglaterra el lombrosismo, que apoyan personalidades de la calidad de Henry Maudsey, se basará en el degeneracionismo moreliano tanto como en el evolucionismo darwiniano (43). También el interés en algunas enfermedades, como la epilepsia, permite dar apoyo a las nuevas doctrinas criminalísticas. Al igual que en todo el mundo, el proceso de somatización de la locura fue un punto de apoyo 
importante para que los médicos defendieran la participación de la profesión en los juicios criminales. Esta intervención aumentó durante el siglo XIX, aunque parece no haber sido muy efectiva en cuanto a la defensa del acusado, pues el veredicto final solía ser de responsabilidad y culpabilidad. La rígida moral victoriana tendía a considerar a todos los hombres responsables de sus propios actos, aunque hubiera manifestaciones de enajenación parcial (44).

En España, los psiquiatras fueron sensibles a las novedades lombrosianas. Entre los ya mencionados, hay que recordar la actividad forense y la orientación somaticista de Arturo Galcerán Granés, cuya orientación fuertemente determinista lo emparenta con los nuevos movimientos. También hay cierta cercanía a la escuela lombrosiana en José María Escuder, quien en su análisis del cura Galeote -magnicida cuyo crimen fue fundamental como el de Carnot para los cambios en actitudes y legislación ante el crimen - afirmaba en 1866: «La desproporción de su cráneo y de su cara enseña a todo hombre observador que nos hallamos frente a un degenerado; se dan en él los estigmas de la herencia, y entre ellos no es menor la fealdad que imprime su fisonomía, el prognatismo de la mandíbula inferior, que la asemeja a las razas más bajas de la escala humana... (45)». De todas formas, al parecer el mismo Escuder desconfía de la importancia de los estigmas y, en general, la medicina española hizo evolucionar el lombrosismo hacia la antropología y la sociología. La obra de Rafael Salillas es muy importante en este sentido y, aunque su libro La vida penal en España (1888) fue muy elogiado por Lombroso, pronto se dirigió hacia un análisis antropológico y sociológico de la marginación. De todas formas, su labor médico-legista, así como la de Olóriz, son deudoras de la escuela italiana (46).

\section{Conclusión: Cuerpos y almas}

Es evidente que al llegar a la segunda mitad del XIX el manejo del enfermo mental iniciado en la Ilustración tocaba a su fin. Hasta mediar el siglo que nos ocupa siguieron rigiendo normas de protección asilar y tratamiento moral. Era importante la salvaguardia de la libertad del enfermo, su reincorporación al mercado de trabajo y un trato apoyado en la nueva ética burguesa. El enfermo se había favorecido, en su adaptación a la nueva sociedad, de una consideración más benéfica y de un esfuerzo por adaptarlo al mercado de trabajo y al orden social (47). Pero el hacina- 
miento y cronificación de enfermos mentales en muchos hospitales europeos llevó poco a poco a perder la confianza que en ellos se tenía. Si a lo largo de todo el siglo las dudas fueron en aumento, será a principios del siglo XX cuando posiciones claras y enfrentadas al sistema asilar dieron paso a las nuevas corrientes críticas. En este sentido, es muy significativa la publicación en París en 1903 por Paul Sérieux de su Rapport sur l'assistance des aliénés en France, en Allemagne, en Italie et en Suisse (48).

La psiquiatría europea finisecular, dominada aún por el degeneracionismo, favoreció un nihilismo terapéutico que trajo dos consecuencias de largo alcance. En primer término, la degradación sistemática y progresiva de los asilos ya que, al colocar al loco en el más rígido de los biologismos y asumirse sin dificultad su incurabilidad, los establecimientos a ellos destinados se convirtieron en "depósitos» de individuos «irrecuperables», abandonados desde el punto de vista médico y administrativo y, condenados, en la mayoría de las ocasiones, al encierro permanente. En segundo lugar, la aparición de especialistas no alienistas que aportaron un saber, para ellos suficiente, para intentar el tratamiento de un buen número de enfermos a los que la medicina mental había desahuciado. Nos referimos, por un lado a los neurólogos, que empezaban a ejercer su nueva y pujante especialidad en hospitales generales y universitarios, o en consultas privadas alejadas del asilo, al que no dudaron en lanzar sus más duras críticas (49); por otro, a cirujanos y ginecólogos quienes, en general, llegaron a proponer agresivas y mutilantes intervenciones para tratar desarreglos mentales diversos (50).

Eran, sin duda, dos buenos motivos para que cundiese la inquietud en el seno del movimiento alienista amenazado de perder sus tradicionales competencias y con una deplorable imagen ante la opinión pública. La búsqueda de alternativas asistenciales será, a partir de entonces, una constante. Por un lado, se intentarán reformas en las políticas sanitarias, como los servicios de puertas abiertas (51), destinadas a superar el manicomio cerrado. Por otro, muchos alienistas se encaminaron hacia una orientación neurológica de su especialidad, a la que los nuevos descubrimientos de la fisiología y la patología del sistema nervioso invitaba. Se ofrecía una nueva vía ante el fracaso del non-restraint que no era capaz de curar muchos casos en los que la causa moral era grave, o bien había que localizar causas orgánicas. Por otro lado, el origen orgánico de la lesión no implicaba de forma contundente la incurabilidad de la enfermedad mental, ni siquiera cronicidad obligada, aunque sí más probable, como afirmó explícitamente Maudsley. 
Es evidente que el tratamiento médico progresaba y que los médicos, al compás de su acercamiento al somaticismo, siguieron esta esperanzadora vía. El citado Maudsley, si bien dudando de la eficacia de muchas drogas, recomienda el tratamiento precoz y el estudio físico y familiar del paciente. Era una vía más hacia el futuro, que se combinaba con el rechazo al encierro asilar. "For the reasons adduced, I cannot but think that the future progress in the improvement of the treatment of the insane lies in the direction of lessening the sequestration and increasing the liberty of them» (52).

\section{NOTAS}

(1) Dados nuestros estudios anteriores, nos centraremos en el occidente europeo, concretamente en España, Gran Bretaña, Francia y parcialmente en Italia.

(2) G. LANTERI-LAURA (1972). «La chronicité dans la psychiatrie française moderne», Annales ESC 27, pp. 548-568; M. FouCAULT (1979), Historia de la locura en la época clásica, 2. ${ }^{\text {a }}$ ed., 2 vol., Madrid; A. Scull (1981), Madhouses, Mad-doctors and Madmen, Pennsylvania; W. Parry-Jones (1972), The Trade in Lunacy, London; R. CANOSA (1979), Storia del manicomio in Italia dall'Unità a oggi, Milán.

(3) J. L. Peset y R. Huertas (1986), «Del 'ángel caído' al enfermo mental: Sobre el concepto de degeneración en las obras de Morel y Magnan", Asclepio 38, pp. 215-240; R. Huertas (1987), Locura y degeneración. Psiquiatría y sociedad en el positivismo francés, Madrid.

(4) J. L. y M. PESET (1975), Cesare Lombroso y la escuela positivista italiana, Madrid.

(5) La eugenesia, movimiento social basado en una doctrina pseudocientífica elaborada por Francis Galton, en la línea de los nacientes positivismo y evolucionismo, buscaba combatir la "degeneración» de la raza, en la que mucho tenían que ver los "débiles mentales» de todo tipo, para los que se piensa en medidas de control de la procreación. Se puede consultar en relación con el origen de la eugenesia, R. ALvarez (1985), Sir Francis Galton, padre de la Eugenesia, Madrid; y para su desarrollo y evolución en España y América Latina: R. Alvarez (1988), "Origen y desarrollo de la eugenesia en España», en J. M. Sánchez Ron (ed.), Ciencia y sociedad en España, Madrid y R. Alvarez (1988), «Eugenesia y control social», Asclepio 40 (2), pp. 29-88.

(6) M. Foucault y otros (1973), Moi, Pierre Rivière..., París, J. L. Peset (1983a), "La sociedad enferma: algunas reflexiones sobre la muerte del presidente Carnot», Asclepio 35 , pp. 177-188.

(7) Como es bien sabido, la nueva forma en el trato de los enfermos mentales que fue surgiendo en algunos países de Europa -Francia e Italia por lo menos- tiene su fecha significativa en Inglaterra con el establecimiento del York Retreat en 1792 y con la publicación, por su autor, de una descripción del establecimiento y su forma de funcionamien- 
to en 1813. El «quitar las cadenas» y dar mejor trato a los alienados, se fue convirtiendo en una nueva forma de tratamiento, y se fue extendiendo en Inglaterra hasta ser aceptado como, prácticamente, la actitud terapéutica básica, que, por otra parte, debía aplicarse en los asilos o instituciones de internamiento.

Con respecto al tratamiento moral y su significación sociológica, es interesante el artículo de Andrew T. Scull (1979), "Moral treatment reconsidered: some sociological comments on an episode in the history of Bristish Psychiatry", Psychological Medicine 9, pp. 421-428.

(8) El registro oficial de médicos no se establecerá hasta 1858.

(9) En 1884 se produce un importante informe de los Metropolitan Commissioners sobre las condiciones en los asilos (County Asylums, Public Lunatic Hospitals, Licensed Madhouses e incluso Workhouses). Se establece en el informe la naturaleza de la insania y su clasificación, así como la opinión que inspira el non-restraint y otros puntos. El informe dará lugar a las leyes de 1845, en que se instituyen, en lugar de los Metropolitan Commissioners, los Lunacy Commissioners, cinco políticos, tres legistas y tres médicos.

Para evaluar el éxito pensemos que se clasificaban como insanos personas con desviaciones laborales o sexuales y que la curación se consideraba producida con la reincorporación al trabajo. Sin embargo, Conolly en Indications of Insanity ya indica la necesidad de distinguir bien unos y otros procesos y no creer que todo comportamiento extraño implica insania.

(10) Así Maudsley se queja de los "social prejudices» y de los cambios en la legislación, "the tendency of recent lunacy legislation, which has suffered not a little from popular panic and professional philantropy", en The Physiology and Pathology of Mind, 2. ${ }^{\mathrm{a}}$ ed., 1868, p. 492.

(11) M. Granville (1877), The Care and the Cure of the Insane, Lancet Commission, London.

(12) En su número de 5 de abril de 1877 The Times decía: «if lunacy continues to increase as at present, the insane will be the majority, and, freeing themselves, will put the sane in asylums". Tomado de K. Jones (1972), A History of the Mental Health Services, London, p. 167

(13) Laurence J. Ray (1981), "Models of Madness in Victorian Asylum Practice», Archives Européenes de Sociologie 22, pp. 229-264.

(14) F. Leuret (1840), Du traitement moral de la folie, París, p. 1. Véase R. Huertas (1988), "Asilos para locos: terapéutica mental y política sanitaria», Asclepio 40(2), pp. 131-150.

(15) En los últimos tiempos, la consideración de la enfermedad mental ligada exclusivamente a un sustrato orgánico justificador de dosis mantenidas de psicofármacos, electrochoques o choques insulínicos, ha quedado radicalmente enfrentada a concepciones psicologistas, de corte dinámico, consideradas más avanzadas. No deja de resultar esclarecedor, sin embargo, el hecho de que el tratamiento moral propuesto por F. LAURET, con sus intentos intimidatorios, pueda considerarse un antecedente histórico de los descondicionamientos agresivos y de las terapias comportamentales que el conductismo viene proponiendo hace tiempo.

(16) E. Goffman (1961), Asylums. Essays on the Social of Mental Patients and other Inmates, Londres.

Asclepio-Vol. XLV-2-1993 
(17) J. M. Comelles (1988), La razón y la sinrazón. Asistencia psiquiátrica y desarrollo del Estado en la España contemporánea, Barcelona.

(18) Para España es muy significativo de este intento de aproximación a otros ámbitos el libro de C. JuARros (1916), La psiquiatría del médico general, Madrid.

(19) G. Swain (1977), Le sujet de la folie, París; M. Gauchet y G. Swain (1980), La pratique de l'esprit humaine. L'institution asilaire et la révolution démocratique, París. J. L. P.ESET (1993), Las heridas de la ciencia, Valladolid.

(20) P. Lain Entralgo (1950), La historia clínica, Madrid; M. Foucault (1963), La naissance de la clinique, París; J. L. PESET (1982), «El principio de causalidad en Medicina», en Escritos en homenaje a Juan Peset Aleixandre, Valencia, 1982.

(21) S. RAMÓN Y CAJAL (1899-1904), Textura del sistema nervioso del hombre y de los vertebrados, 3 vols., Madrid.

(22) J. SCHILlER (1967), Claude Bernard et les problèmes de son temps, París, 1967; y P. Lain Entralgo (1947), Claudio Bernard, Madrid.

(23) Desarrollaron el método analítico Pinel y Cabanis. Véase J. M. LoPEz PIÑERo y J. M. Morales Meseguer (1970), Neurosis y psicoterapia, Madrid y E. Arouiola (1990), «La aplicación del método analítico al estudio de la enfermedad en Francia en el tránsito del siglo XVIII al XIX». Asclepio 42 (I), 237-251; E. Arquiola, L. MonTIEL (1993), La corona de las ciencias naturales, Madrid, C.S.I.C.

(24) V. Magnan y P. M. Legrain (1895), Les dégénérés. État mental et syndromes épisodiques, París, p. 74.

(25) R. CASTEL (1976), L'ordre psychiatrique, París.

(26) Consultar, para la historia de este proceso de transición, el excelente trabajo de RoBert M. Young (1968), "The Functions of the Brain: Gall to Ferrier (1808-1886)", Isis 59, pp. 251-258. Para la posterior obra de Broca, Elvira Arouiola (1976), «Paul Broca y la antropología positivista francesa", Asclepio 28, 51-92 y F. Schiller (1990), Paul Broca, Explorateur du cerveau, París.

(27) Para una visión general de la psiquiatría inglesa entre la Restauración y la Regencia es muy útil la obra de Roy PorTER (1967), Mind-forget Manacles. A History of Madness in England, Cambridge, Massachussets. Para el tema que nos ocupa, véase el capítulo 4. "The Making of Psychiatry», p. 169.

(28) L. S. JACYNA (1982), "Somatic Theories of Mind and the Interests of Medicine in Britain, 1850-1879», Medical History 26, 233-258, p. 235.

(29) A. BAIN (1872), Mind and Body, London, 1872, p. 196. «The argument for the two substances have, we believe, now entirely lost their validity: they are no longer compatible with ascertained science and clear thinking. The one substance with two sets of qualities the physical and mental - a double facet unity - would comply with all exigencies of the case».

(30) Las relaciones mente-cuerpo podían ser concebidas como relaciones entre dos sistemas diferentes, conservando el dualismo de origen cartesiano, que mantenían relaciones o interrelaciones más o menos directas. Jackson optó por una concepción de sistemas paralelos, de forma que podía estudiar uno sin preocuparse del otro; podía estudiar los fenómenos cerebrales sin preocuparse de los problemas metafísicos inherentes al estudio de las facultades mentales. En otros casos se aceptaba una relación más estrecha, una dependencia de las facultades con respecto a las funciones nerviosas. Y, en general, se ter- 
minó por aceptar la existencia de funciones y no de facultades. Las posturas más extremas, como la mantenida por Henry Maudsley, consideraba que toda actividad mental, consciente o insconsciente, era una actividad nerviosa.

Sobre la posición de Jackson, J. M. Lopez PIÑERo (1973), John Hughlings Jackson (1835-1911), Madrid.

(31) Los trabajos de Marshall Hall sobre la fisiología de la función refleja, y los de Carpenter y Thomas Laycock fueron fundamentales en cuanto a la comprensión de la función fisiológica cerebral. HUGHLINGS JACKSON en la clínica y DAVID FERRIER en la experimentación animal, fueron algunos de los grandes estudiosos ingleses del problema de la localización de funciones.

(32) D. H. TuKe (1881), «Presidential Address, delivered at the Annual Meeting of the Medico-Psychological Association, held at the University College», The Journal of Mental Science 27, n. ${ }^{\circ} 119$, pp. 305-342.

(33) J. C. Bucknill, D. H. Tuke (1879), A Manual of Psychological Medicine, 4th ed., London, 1879, pp. 493 y vii.

(34) Sobre Luis Simarro véanse los trabajos de Agustin Albarracin y de Jose Sala (1978), en J. Campos y R. Llavona (ed.). Los orígenes de la psicología científica en España: el doctor Simarro, en Investigaciones psicológicas 4, pp. 99-113 y 83-107.

(35) S. Ramón y Cajal (1890), Manual de Anatomía Patológica General, Madrid, pp. 5-6.

(36) A. GALCERÁN (1881), "El proceso de la razón», Revista Frenopática de Barcelona $1,88-102$

(37) A. Galcerán (1881), "Causas próximas de la locura o Anatomía Patológica cerebral», Revista Frenopática de Barcelona 1, 248-257. También se observa influencia del degeneracionismo, al menos en la importancia que concede a la herencia. Véase J. B. Ullersperger (1954), Historia de la psiquiatría y de la psicología en España, Madrid, así como E. Domenech y J. CoRbella (1969), «La obra psiquiátrica de Arturo Galcerán Granés", Actas del III Congreso Nacional de Historia de la Medicina, Valencia, I, pp. 143-157.

(38) En el futuro también habrá un camino hacia la «espiritualización» de la culpa, que irá hacia el psicoanálisis. celona.

(39) J. L. y M. Peset (1975), Madrid; J. L. Peset (1983b), Ciencia y marginación, Bar-

(40) Ch. FÉRÉ (1888), Dégénérescence et criminalité, París, pp. 56-57.

(41) Se trata de un trabajo presentado en el III Congreso de Antropología Criminal celebrado en la capital belga en 1892, véase V. MAGNAN (1892), L'obsession criminelle morbide, Bruxelles.

(42) Relaciona la fuerte evolución social y sus problemas - anarquismo, militarismo, diferencias sociales, injusticias-con la más lenta evolución del ser humano concreto "Le cerveau humaine est un et son perfectionnement est si lent qu'il est presque immuable comme les espèces animales. Il y a dans le cerveau certains instincts essentiels, inéluctables, primoriaux, que rien n'a encore modifiés et qui ne changeront pas plus que les membres et le corps de l'homme». A. Lacassagne (1894), L'Assassinat du Président Carnot, Lyon-París, p. 9; véase también de este autor, L'homme criminel comparé à l'homme primitif, Lyon, 1882, pp. 15-16 y 20. Véase J. L. PESET (1983a).

(43) H. MAudsley (s.a.), El crimen y la locura, Valencia, "Además -es otro fenómeno observado- la clase criminal constituye una variedad degenerada y morbosa de la 
especie humana, marcada por caracteres particulares de inferioridad física y mental», p. 35.

(44) J. L PESET (1983b), 96 ss y 191 ss.

(45) En J. M. Escuder (1895), Locos y anómalos, Madrid, citado por A. GALERA, «La antropología criminal en España: su proceso de asimilación y evolución", Asclepio 39(1), pp. 273-289, p. 276. Sobre este caso, véase J. VArela y F. Alvarez Uría (1979), El cura Galeote asesino del obispo de Madrid-Alcalá, Madrid.

(46) A. GALERA (1986), «Rafael Salillas: medio siglo de antropología criminal española", Llull 9, pp. 81-104. También el mundo americano conoció la escuela lombrosiana, véase A. GALERA (1988), "El resurgir de una nueva escuela: Israel Castellanos y el atavismo del delito", Asclepio 40(2), pp. 81-97; Ciencia y delincuencia, Madrid, C.S.I.C., 1991. También J. L. PEset (1983b), tercera parte.

En este terreno se debe citar al español Lecha Marzo, véase J. MartinEz (1986), «La profilaxis médica del crimen en la obra de Lecha Marzo", Asclepio 38, pp. 241-272.

(47) R. CASTEL, L'ordre psychiatrique, y K. DöRnER (1974), Ciudadanos y locos. Historia social de la psiquiatría, Madrid; M. Alvarez URIA (1983), Miserables y locos, Barcelona y R. HuerTas (1990), "Medicina mental y revolución burguesa: sobre los orígenes de la asistencia psiquiátrica en Francia», Revista de la Asociación Española de Neuropsiquiatría, 10, 389-399.

(48) Sobre intentos alternativos al manicomio en el siglo pasado, véase R. HUERTAS (1989), "La comunidad de Gheel: una alternativa de asistencia psiquiátrica en el siglo XIX», Revista de la Asociación Española de Neuropsiquiatría 8, 257-266.

(49) En USA, por ejemplo, se produjo una interesante lucha "corporativa» entre ambas especialidades, que ha sido estudiada por J. M. QuEN (1977), "Asylum, Psychiatry, Neurology, Social Work and Mental Hygiene: an Exploratory Study in Interprofessional History", Journal of the History of the Behavioral Sciences 13, pp. 3-11.

(50) Sobre estos temas véase A. Scull, D. FaVrear (1987), "Médecine de la folie ou folie des médecins? Controverse à propos de la chirurgie sexuelle au 19e siècle», Actes de la Recherche en Sciences Sociales 68, pp. 31-41. También para Inglaterra Eduardo BaLBo (1990), "La mujer, la neurofisiología y la locura», Asclepio 42(2), 137-160. Para Italia véase el excelente libro de VALERIA P. BABINI et al. (1986), La donna nelle science dell'uomo, Milano.

(51) R. Huertas (1989), "Edouard Toulouse y el servicio psiquiátrico de "puertas abiertas" ", Asclepio 41(1), 261-280. También R. HuERTAS (1992), Del manicomio a la salud mental. Para una historia de la Psiquiatría Pública. Madrid.

(52) H. Maudsley (1868), The Physiology and Pathology of Mind, London, p. 501. Tuke también se mantiene en las dudas, cree en el futuro del tratamiento aunque duda de su efectividad absoluta. Pero es interesante que el progreso del tratamiento lo relacione con las enfermedades orgánicas: "Remedies, like iodide of potassium, in large doses, are employed in cases which, from the increased attention directed in recent years to the somatic aetiology of insanity, a casual relation between the physical and mental condition was recognized and the mental symptoms have disappeared in the most marked manner", en D. H. TuKE «Presidential Address», p. 333. Señala que hay casos que se curan, a diferencia de cuarenta años antes, señalando la importancia del desarrollo de tratamientos específicos. 